\title{
Engagement académico e Inteligencia Emocional en adolescentes
}

\author{
Begoña María Tortosa, María del Carmen Pérez-Fuentes, María del Mar \\ Molero, José Gabriel Soriano, Nieves Fátima Oropesa, María del Mar Simón, \\ María Sisto, y José Jesús Gázquez \\ Universidad de Almería
}

El compromiso por los estudios, la dedicación y la satisfacción académica, entre otras variables, pueden ser desencadenantes del engagement, un estado positivo vinculado con el estudio o trabajo. Sin embargo, si prestamos atención a los adolescentes, existen otras variables que pueden preceder al engagement académico. Desde hace unos años, hay una gran cantidad de investigadores que han mostrado una tendencia a estudiar el tema del engagement académico y la Inteligencia Emocional (IE) debido a su relevancia. Por este motivo, la presente revisión pretende analizar la investigación previa sobre la relación entre estas variables. El objetivo principal es analizar si existe o no relación entre las variables de engagement académico e Inteligencia Emocional en adolescentes. Los estudios revisados muestran resultados que indican la existencia de correlaciones entre las variables de engagement académico e IE en los adolescentes. Finalmente, hay que hacer hincapié en la relación existente entre el engagement académico y la inteligencia emocional para conseguir que los adolescentes desarrollen habilidades emocionales y, consecuentemente, se sientan satisfechos académicamente.

Palabras clave: Engagement académico, Inteligencia emocional, adolescentes.

Commitment to studies, dedication and academic satisfaction, among other variables, can trigger engagement, a positive state linked to study or work. However, if we pay attention to adolescents, there are other variables that can precede academic engagement. For a few years, there are a lot of researchers who have shown a tendency to study the topic of academic engagement and Emotional Intelligence (EI) because of its relevance. For this reason, this review aims to analyze the previous research on the relationship between the variables. The main objective is to analyze whether or not there is a relationship between the variables of academic engagement and emotional intelligence in adolescents. Studies show results which point the existence of correlation between the variables of academic engagement and EI in adolescents. Finally, emphasis must be placed on the relationship between academic engagement and emotional intelligence in order to ensure that adolescents develop emotional skills and, consequently, feel satisfied academically.

Keywords: Academic engagement, Emotional intelligence, adolescents.

\footnotetext{
Correspondencia: María del Carmen Pérez Fuentes. Departamento de Psicología Evolutiva y de la Educación. Universidad de Almería. Ctra. La Cañada de San Urbano s/n, C.P. 04120. Almería (España). E-mail: perezfuentes@ual.es
} 
Durante la etapa adolescente se producen un gran número de situaciones personales y ambientales que influyen significativamente en los jóvenes, tanto en su formación como en su desarrollo personal. Puesto que la adolescencia es una etapa de transición hacia la edad adulta en la que se experimentan cambios en la vida de la persona (Gómez-Fraguela, Fernández, Romero, y Luengo, 2008). Algunos estudiantes no desarrollan ciertas estrategias y competencias que son esenciales para las dificultades de la vida académica, pudiendo tener actitudes negativas como desinterés por los estudios, dudas sobre la capacidad personal, agotamiento, desmotivación y abandono de los estudios. Estas características se ocupan bajo el síndrome de burnout académico (Palacio, Caballero, González, Gravini, y Contreras, 2012). Según Usán, Salavera, y Domper (2018), el burnout académico se conoce por un patrón conductual desadaptativo.

Sin embargo, no siempre los jóvenes experimentan sensaciones negativas, sino que algunos alumnos/as, cuando hablan de estudios, sienten entusiasmo, dedicación, interés, energía, resistencia a la demanda escolar, persistencia ante las dificultades, compromiso, y absorción (Schaufeli y Bakker, 2004). Cuando aparecen estas manifestaciones podemos hablar de engagement académico.

El engagement académico es definido como un "estado mental positivo relacionado con el trabajo y caracterizado por vigor, dedicación y absorción” (Schaufeli, Salanova, González-Romá, y Bakker, 2002, p. 79). Por otro lado, se define como un patrón de conducta adaptativo y caracterizado por el desempeño de las funciones académicas, la implicación y la complacencia hacia los estudios. Es por esto que las características del burnout académico se oponen a las del engagement (Usán et al., 2018).

Además, el engagement académico se relaciona con una alta autoeficacia (Bresó, Schaufeli y Salanova, 2011), un rendimiento académico excelente (Vera, Le Blanc, Taris, y Salanova, 2014), inteligencia emocional (Extremera, Durán, y Rey, 2007) y alta satisfacción de los estudiantes (Moyano y Riaño, 2013).

El concepto de IE se ha utilizado a mediados de los años 90, prestando una especial atención a las emociones.

Algunas de las investigaciones ponen de manifiesto la importante relación entre el engagement académico y la inteligencia emocional (Extremera et al., 2007).

Esta revisión se centrará en la Inteligencia Emocional (IE), la cual ha sido definida por diversos autores:

Según Sternberg (1997), se define la Inteligencia Emocional como la capacidad del individuo de adaptación. Se relaciona con las emociones, el optimismo, la memoria, la salud mental y la creatividad (Trujillo, 2005).

Para Mayer, Salovey, y Caruso (2000), la IE se define como una capacidad mental para expresar, regular y entender las emociones con precisión, una capacidad para producir sentimientos que provoquen el pensamiento, una seña de personalidad y un movimiento de la cultura (Fernández, 2013; Serrano y Andreu, 2016). 
La IE es un aspecto relevante a la hora de adaptarnos al ambiente que nos rodea y para tener éxito. Igualmente, la IE es una habilidad para procesar la información sobre emociones. Es por este motivo por el que adquiere una especial relevancia dentro de la educación, a través de la llamada educación emocional (Fernández, 2013).

En este sentido, Serrano y Andreu (2016) afirman que las capacidades emocionales desarrolladas gracias a la Inteligencia Emocional, al favorecer un mejor entendimiento y procesamiento de la información emocional, influyen en el bienestar, en el equilibrio psicológico y/o en una práctica académica favorable. Así como, en las conductas de dedicación, interés, compromiso, y absorción. Es decir, en el engagement académico.

Por otro lado, los trabajos de Rey, Extremera, y Pena (2011) han demostrado evidencias empíricas de que existe una relación positiva entre IE y las dimensiones que caracterizan el engagement según Shaufeli et al. (2002): vigor, dedicación y absorción.

Gracias a esta información y siguiendo a Usán et al. (2018), es de esperar que haya cada vez más investigaciones y estudios acerca de la relación entre la Inteligencia Emocional y el engagement académico, con el objetivo de analizar y comprender dicha relación.

\section{Objetivo}

Comprender y analizar si existe o no relación entre las variables de engagement académico e Inteligencia Emocional en adolescentes.

\section{METODOLOGÍA}

\section{Estrategia de búsqueda}

Para la realización de esta revisión sistemática se procedió a efectuar una búsqueda bibliográfica previa en bases de datos científicas relacionadas con las ciencias de la educación, la psicología, las ciencias sociales y de la salud. Entre ellas se encuentran: Dialnet, Web of Science, Scopus y Scielo.

Tabla 1. Fórmulas de búsqueda

\begin{tabular}{lcc}
\hline $\begin{array}{l}\text { Bases de } \\
\text { datos }\end{array}$ & Descriptores & Fórmulas de búsqueda \\
\hline Dialnet & $\begin{array}{c}\text { Engagement académico, Inteligencia } \\
\text { Emocional y adolescentes }\end{array}$ & $\begin{array}{c}\text { Engagement académico y adolescentes, Inteligencia } \\
\text { Emocional y Engagement académico }\end{array}$ \\
\hline $\begin{array}{l}\text { Web of } \\
\text { Science }\end{array}$ & $\begin{array}{c}\text { Engagement académico, Inteligencia } \\
\text { Emocional y adolescentes }\end{array}$ & $\begin{array}{c}\text { Engagement académico y adolescentes, Inteligencia } \\
\text { Emocional y Engagement académico }\end{array}$ \\
\hline Scopus & $\begin{array}{c}\text { Engagement académico, Inteligencia } \\
\text { Emocional y adolescentes }\end{array}$ & $\begin{array}{c}\text { Engagement académico y adolescentes, Inteligencia } \\
\text { Emocional y Engagement académico }\end{array}$ \\
\hline Scielo & $\begin{array}{c}\text { Engagement académico, Inteligencia } \\
\text { Emocional y adolescentes }\end{array}$ & $\begin{array}{c}\text { Engagement académico y adolescentes, Inteligencia } \\
\text { Emocional y Engagement académico }\end{array}$ \\
\hline
\end{tabular}


En primer lugar, para la búsqueda, se usaron los siguientes descriptores: "Engagement académico" en español y en inglés, "inteligencia emocional" y "adolescentes", y los operadores booleanos fueron "AND".

En segundo lugar, se usaron las siguientes fórmulas de búsqueda: "engagement académico AND adolescentes" e "inteligencia emocional AND Engagement" (Tabla 1).

Los descriptores "engagement", "adolescentes" e "inteligencia emocional" se aplicaron al título, al resumen o a las palabras clave de las publicaciones encontradas, con el objetivo de evitar trabajos que estudiaran otros campos diferentes a los estudiantes adolescentes.

\section{Criterios de inclusión y exclusión}

Con la finalidad de conseguir información precisa y actual, el proceso de elección de las publicaciones se ha filtrado siguiendo unos criterios: el argumento del resumen, la relevancia y utilidad para la investigación, si el tema de estudio se centra en adolescentes, el año de publicación y el idioma (inglés y español).

Como criterios para la inclusión de trabajos se usaron aquellas publicaciones: a) que la finalidad del estudio fuera la relación entre el engagement académico y la Inteligencia Emocional, cuya muestra se centrara en adolescentes. Los estudios tienen que estudiar al menos una de las variables, b) que tuvieran acceso al texto completo, c) que fueran artículos científicos evaluados siguiendo unos criterios, d) se incluyeron publicaciones empíricas en cuyo título o resumen se encontrara el principal descriptor "engagement", acompañado de algunos de los descriptores mencionados y e) que los trabajos estuvieran escritos en inglés o en español.

Los criterios de exclusión de trabajos han sido: a) publicaciones cuyo resumen no se basará en el objetivo de la revisión, b) aquellas publicaciones redactadas en un idioma diferente al español o al inglés, c) se exceptuaron los documentos anteriores a 2004, d) artículos que no tenían acceso al texto completo, e) publicaciones cuya muestra no estuviera formada por adolescentes y f) aquellos trabajos que sean notas editoriales, tesis doctorales y aportaciones a congresos.

\section{Recolección de datos}

En la búsqueda inicial en las bases de datos explicitadas con anterioridad, gracias a los descriptores y las fórmulas de búsqueda, se encontraron un total de 32 documentos, prestando atención a los criterios de inclusión y exclusión. Los documentos se dividieron de la siguiente manera: Dialnet (26), Web of Science (1), Scopus (3) y Scielo (2).

Tras aplicar los criterios de exclusión e inclusión y los filtros en cada una de las bases de datos, se tuvo en cuenta la duplicación de los artículos en las diversas bases de datos: Dialnet (23), Web of Science (0), Scopus (1) y Scielo (1), quedando un total de 25 
artículos. Seguidamente, al exceptuar los documentos cuyo título y resumen no se adaptaban a la muestra a analizar, al idioma o a la línea de investigación, quedaron en Dialnet (8), Web of Science (0), Scopus (1) y Scielo (0), reultando un total de 9 artículos. Eliminando los estudios sin acceso al texto completo, se dejaron en Dialnet (8), Web of Science (0), Scopus (0) y Scielo (0), con un total de 8 documentos. Finalmente, se excluyeron los trabajos escritos en otro idioma diferente al español y al inglés (portugués) y aquellos que no relacionaban directamente las variables de estudio, quedando 6 artículos que cumplían los criterios de selección previstos (Figura 1).

Figura 1. Diagrama de flujo con el proceso de selección de artículos

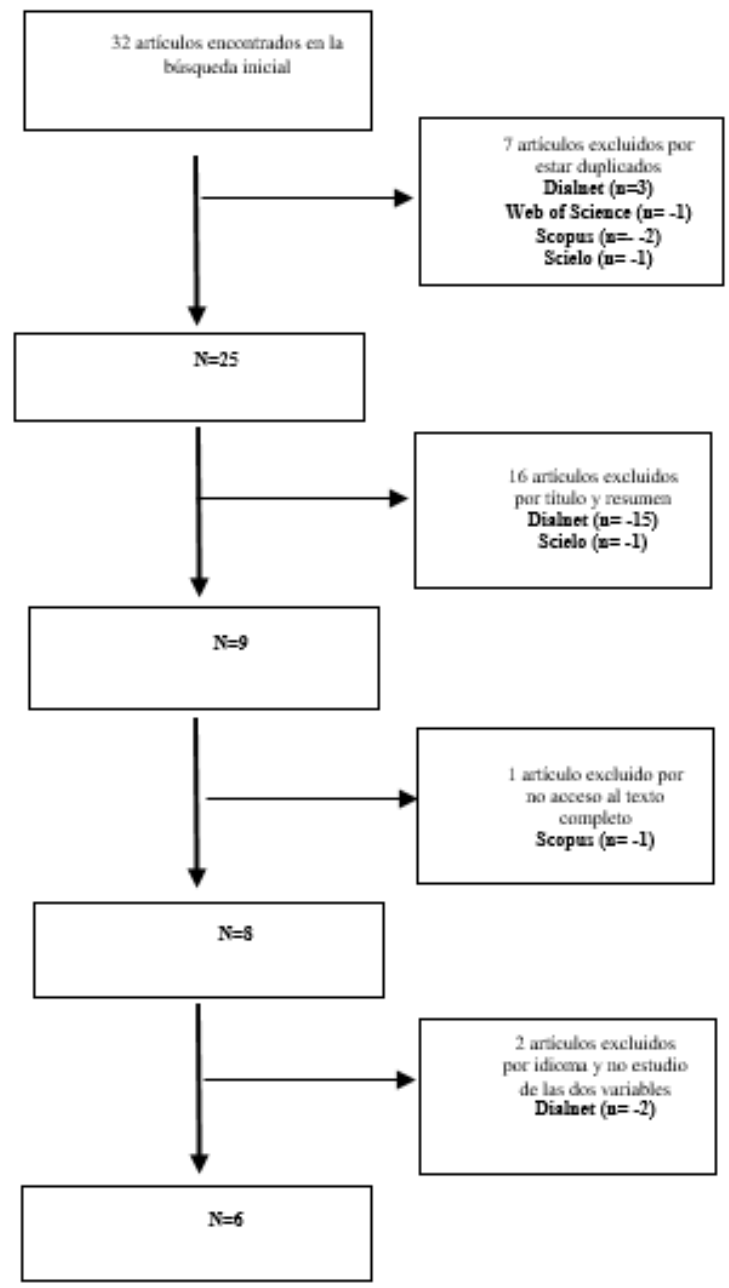




\section{RESULTADOS}

Las variables estudiadas en los trabajos revisados fueron distribuidas en 4 grupos, según su naturaleza. La totalidad fue de 11 factores que quedaron organizados en las siguientes variables: sociodemográficas, contextuales, sociales y psicológicas. La división de estas variables y factores se muestra en la Tabla 2.

Tabla 2. Variables estudiadas

\begin{tabular}{lc}
\hline Variables estudiadas & Factores $\mathrm{y}^{\circ}$ de artículos \\
\hline Sociodemográficas & Género (1), Edad (1) \\
\hline Contextuales & Tipo de centro (1) \\
\hline Sociales & Clima escolar (1), Apoyo docente (1), \\
\hline \multirow{2}{*}{ Psicológicas } & $\begin{array}{r}\text { Inteligencia Emocional (1), Autoeficacia (1), Compromiso escolar (1), } \\
\text { Motivación (1), Satisfacción (1), Motivación (1) }\end{array}$ \\
\hline
\end{tabular}

En la Tabla 3, se muestran las características generales de los artículos analizados. Además, se contemplan los autores, instrumentos para medir el engagement académico y las variables estudiadas.

Tabla 3. Características de los artículos

\begin{tabular}{|c|c|c|}
\hline Autor/es (año) & Variables & Instrumentos \\
\hline $\begin{array}{l}\text { Tomás, Gutiérrez, } \\
\text { Sancho, Chireac, } \\
\text { y Romero (2016) }\end{array}$ & $\begin{array}{c}\text { Sociodemográficas, contextuales, sociales } \\
\text { y psicológicas. }\end{array}$ & $\begin{array}{c}\text { Escalas de Likert (de 1 a 5): } \\
\text {-Versión en español de la E. Compromiso Escolar (Veiga, } \\
\text { 2013). } \\
\text {-E. unidimensional de Compromiso Escolar (Nie y Lau, } \\
\text { 2009). } \\
\text {-E. Satisfacción con la Escuela (Nie y Lau, 2009). } \\
\text {-Orientación de metas y Clima Motivacional en el Aula } \\
\text { (Stornes y Bru, 2001). } \\
\text {-Apoyo a la Autonomía (Jang, Kim, y Reeve, 2012). }\end{array}$ \\
\hline $\begin{array}{l}\text { Gutiérrez, Tomás, } \\
\text { Barrica, y Romero } \\
\text { (2017) }\end{array}$ & Contextuales, sociales y psicológicas. & $\begin{array}{c}\text { Escalas de Likert (de 1 a 5): } \\
\text { A) Para el clima motivacional }{ }^{1} \text { del aula: } \\
\text { 1. Cuestionario de orientación y clima motivacional } \\
\text { (Stornes y Bru, 2011). } \\
\text { 2. Escala de satisfacción de las necesidades psicológicas } \\
\text { básicas (Sheldon y Hilper, 2012). } \\
\text { 3. Cuestionario de percepción de apoyo a la autonomía del } \\
\text { alumno (Jang et al., 2012). } \\
\text { B) Para el engagement: } \\
\text { 4. School Engagement Scale-SES-4D (Veiga, 2013). } \\
\text { C) Para el logro académico: } \\
\text { 5. Cuestionario de éxito académico (Plunkett et al., 2008). } \\
\text { 6. Calificaciones de idioma y matemáticas del alumno/a } \\
\text { (escala de 0 a 20). }\end{array}$ \\
\hline $\begin{array}{l}\text { Serrano y Andreu } \\
\text { (2016) }\end{array}$ & $\begin{array}{l}\text { Contextuales, sociodemográficas y } \\
\text { psicológicas. }\end{array}$ & $\begin{array}{l}\text { Escalas de Likert (de 1 a 5): } \\
\text { 1. Versión española adaptada del TMMS (Fernández- } \\
\text { Berrocal et al., 2004). } \\
\text { 2. Adaptación española de Subjective Happiness Scale } \\
\text { (Extremera, Fernández, González, y Cabello, 2009). } \\
\text { 3. Versión española adaptada de Perceived Stress Scale } \\
\text { (Cohen, Kamark, y Mermelstein, 1983). } \\
\text { 4. Adaptación española de Utrecht Work Engagement Scale } \\
\text { for Students (Benavides, Fraiz de Camargo, y Porto, 2009). }\end{array}$ \\
\hline
\end{tabular}


Tabla 3. Características de los artículos (continuación)

\begin{tabular}{|c|c|c|}
\hline Autor/es (año) & Variables & Instrumentos \\
\hline $\begin{array}{l}\text { Veiga, García, } \\
\text { Reeve, Weantzel, } \\
\text { y García (2015) }\end{array}$ & Sociodemográficas y psicológicas. & $\begin{array}{l}\text { 1. Student's Engagement in School (Veiga, 2013). } \\
\text { 2. Versión adaptada al portugués de Piers-Harris Children's } \\
\text { Self-Concept Scale (Rodrigues, Veiga, Fuentes, and García, } \\
\text { 2013). }\end{array}$ \\
\hline $\begin{array}{l}\text { García, } \\
\text { Lorenzino, y Gray } \\
\text { (2019) } \\
\end{array}$ & Sociodemográficas y psicológicas. & 1. School Engagement (CIHI, 2005). \\
\hline $\begin{array}{l}\text { Portolés y } \\
\text { González (2015) }\end{array}$ & Psicológicas. & $\begin{array}{l}\text { Escalas de Likert (de } 1 \text { a 7): } \\
\text { 1. Cuestionario Health Behaviour in School-aged (HBSC, } \\
\text { 2010). } \\
\text { 2. Maslash Burnout Inventory-General Survey (MBI-GS; } \\
\text { Schaufeli et al., 2002). }\end{array}$ \\
\hline
\end{tabular}

En la Tabla 4, se muestran los resultados analizados en los 6 artículos incluidos en la muestra de la revisión sistemática.

García, Lorenzino, y Gray (2019) y Tomás et al. (2016), afirman que el compromiso escolar en la adolescencia está influido por la familia, el propio individuo, los amigos/as, el clima escolar y la sociedad. Por ello, los estudios muestran que los adolescentes que estudian en un buen clima de aprendizaje y tareas, familiar, social, etc., tienen una mayor motivación para aprender y una mayor implicación en procesamientos cognitivos más profundos. Por tanto, García et al. (2019), afirman que, a un mayor nivel de disfrute, los adolescentes obtienen un mayor compromiso y participación y Tomás et al. (2016) verifican que tener engagement y un compromiso afectivo con la escuela, favorece la satisfacción escolar.

Tabla 4. Resultados de la búsqueda sobre Inteligencia Emocional y Engagement académico

\begin{tabular}{|c|c|c|c|c|c|c|}
\hline $\begin{array}{l}\text { Autor/es } \\
\text { (año) }\end{array}$ & Aspectos evaluados & Muestra & $\begin{array}{l}\text { Edad de la } \\
\text { muestra }\end{array}$ & $\begin{array}{c}\text { Características de la } \\
\text { muestra }\end{array}$ & $\begin{array}{l}\text { País de } \\
\text { estudio }\end{array}$ & Resultados \\
\hline $\begin{array}{l}\text { Tomás, } \\
\text { Gutiérrez, } \\
\text { Sancho, } \\
\text { Chireac, y } \\
\text { Romero } \\
\text { (2016) }\end{array}$ & $\begin{array}{c}\text { Compromiso escolar de } \\
\text { adolescentes y aspectos } \\
\text { vinculados. }\end{array}$ & $\begin{array}{c}3172 \text { estudiantes: } \\
* 2034 \text { de } \\
\text { Benguela (1019 } \\
\text { mujeres y } 1015 \\
\text { hombres) } \\
\text { **1138 de Santo } \\
\text { Domingo ( } 662 \\
\text { mujeres y } 476 \\
\text { hombres) }\end{array}$ & $\begin{array}{l}* 17,5 \\
\text { años } \\
* * 15,5 \\
\text { años. }\end{array}$ & $\begin{array}{c}\text { *Viven en áreas } \\
\text { urbanas } \\
* * \text { El } 58.3 \% \\
\text { estudiaban en } \\
\text { institutos públicos, el } \\
22.8 \% \text { en privadas y el } \\
18.8 \% \text { en Formación } \\
\text { Profesional. }\end{array}$ & $\begin{array}{l}\text { *Angola y } \\
\text { **República } \\
\text { Dominicana }\end{array}$ & $\begin{array}{l}\text { - La autonomía y el apoyo } \\
\text { docente fomenta el } \\
\text { compromiso escolar. } \\
\text {-La responsabilidad tiene } \\
\text { correlación con el } \\
\text { compromiso. }\end{array}$ \\
\hline $\begin{array}{l}\text { Gutiérrez, } \\
\text { Tomás, } \\
\text { Barrica, y } \\
\text { Romero } \\
(2017) \\
\end{array}$ & $\begin{array}{c}\text { Percepción del clima } \\
\text { motivacional del aula, } \\
\text { el engagement de los } \\
\text { alumnos/as y el logro } \\
\text { académico. }\end{array}$ & $\begin{array}{c}2028 \\
\text { adolescentes de } \\
\text { Benguela } \\
\text { (Angola) }\end{array}$ & 17,5 años. & $\begin{array}{l}50.1 \% \text { son mujeres. } \\
52.8 \% \text { proceden de } \\
\text { áreas urbanas. }\end{array}$ & Angola. & $\begin{array}{l}\text {-La sensación del clima } \\
\text { motivacional influye de } \\
\text { manera directa con el } \\
\text { engagement de los } \\
\text { adolescentes. }\end{array}$ \\
\hline $\begin{array}{l}\text { Serrano y } \\
\text { Andreu } \\
(2016)\end{array}$ & $\begin{array}{c}\text { Capacidad para } \\
\text { conocer los } \\
\text { sentimientos y } \\
\text { emociones propias, } \\
\text { comprensión de } \\
\text { emociones y habilidad } \\
\text { para alargar en el } \\
\text { tiempo las emociones } \\
\text { positivas. }\end{array}$ & $\begin{array}{l}626 \text { adolescentes } \\
\text { (309 mujeres y } \\
317 \text { hombres) de } \\
\text { Castellón y } \\
\text { Valencia. }\end{array}$ & $\begin{array}{l}\text { De 13-18 } \\
\text { años. }\end{array}$ & $\begin{array}{c}\text { De } 4 \text { institutos } \\
\text { urbanos y laicos: } 3 \\
\text { públicos y } 1 \\
\text { privado/concertado. }\end{array}$ & España. & $\begin{array}{l}\text {-Existe una correlación entre } \\
\text { la Inteligencia Emocional } \\
\text { Percibida y el engagement. }\end{array}$ \\
\hline
\end{tabular}


Tabla 4. Resultados de la búsqueda sobre Inteligencia Emocional y Engagement académico (continuación)

\begin{tabular}{|c|c|c|c|c|c|c|}
\hline $\begin{array}{l}\begin{array}{l}\text { Autor/es } \\
\text { (año) }\end{array} \\
\end{array}$ & Aspectos evaluados & Muestra & $\begin{array}{c}\text { Edad de la } \\
\text { muestra }\end{array}$ & $\begin{array}{c}\text { Características de la } \\
\text { muestra }\end{array}$ & $\begin{array}{l}\text { País de } \\
\text { estudio }\end{array}$ & Resultados \\
\hline
\end{tabular}

Asimismo, Tomás et al. (2016), confirman que existen otras variables diferentes que influyen significativamente en los adolescentes a la hora de tener compromiso escolar. Entre ellas podemos destacar que, un mal comportamiento en la escuela está relacionado con la falta de engagement y que la autonomía y el apoyo del profesorado fomentan el compromiso y la responsabilidad de los estudiantes. Por tanto, las relaciones y la autonomía pronostican el desarrollo afectivo y cognitivo, dando importancia al apoyo que percibe el alumno/a por parte de su profesor (Gutiérrez et al., 2017).

Una curiosidad del estudio de Tomás et al. (2016) es que incluye diferentes nacionalidades. Esto hace que presente un mayor rigor en los resultados y que tenga un carácter transcultural. Los instrumentos usados evalúan aspectos relacionados con el engagement. Entre ellos se encuentran: factores cognitivos, afectivos y conductuales evaluados por la Escala de Compromiso Escolar; sensación del esfuerzo, capacidad de atención y participación en el aula gracias a la Escala unidimensional de Compromiso Escolar; la satisfacción en la escuela, el clima motivacional del centro y las orientaciones del aprendizaje y desempeño, analizado por el instrumento de Orientación de Metas Motivacional en el Aula. Finalmente, con el apoyo a la Autonomía, se estudiaron las sensaciones que tienen los estudiantes en cuanto al apoyo que les brindan sus profesores sobre la autonomía de los adolescentes (Tomás et al., 2016).

Por otro lado, para Gutiérrez et al. (2017), existe una correlación significativa entre la satisfacción de las necesidades psicológicas básicas y la percepción del clima de aprendizaje y engagement. Sin embargo, la correlación entre el clima de aprendizaje y la satisfacción de las necesidades psicológicas básicas con el rendimiento, es baja. Además, 
existe una relación entre el clima de aprendizaje y la satisfacción de las necesidades psicológicas básicas con el apoyo a la autonomía. Así como, hay una correlación más significativa entre el compromiso conductual y las calificaciones, que con la sensación del logro académico. Por ello, según Gutiérrez et al. (2017), el engagement es una demostración de la motivación percibida que tienen los estudiantes de su aprendizaje y, la sensación del clima motivacional por parte de los estudiantes, influye de manera directa con el engagement de los adolescentes.

Si analizamos la relación entre IE y engagement, según los estudios de Serrano y Andreu (2016), encontramos que la inteligencia emocional percibida por los estudiantes no correlaciona con el rendimiento académico. Sin embargo, el engagement académico se relaciona, de una manera positiva, con el propio bienestar y con el rendimiento y, de forma negativa, con el estrés.

Por otro lado, un aspecto a tener en cuenta en el estudio de Serrano y Andreu (2016), es que la edad no tiene relación directa con la comprensión de las emociones, el bienestar, el estrés, la dedicación y el rendimiento académico. Pero, sí se relaciona con el sexo de las personas, puesto que las mujeres tienen mayores notas en dedicación, atención en las emociones, estrés y rendimiento. Mientras que los hombres obtienen mayores puntuaciones en bienestar y reparación de emociones. Concluyendo, para Serrano y Andreu (2016), existe una correlación entre la inteligencia emocional percibida y el engagement. Al igual que entre inteligencia emocional percibida y el estrés o burnout académico.

Al analizar los resultados de Veiga, García, Reeve, Weantzel, y García (2015), se ha verificado que los adolescentes con un alto autoconcepto, poseen un alto engagement (alta capacidad cognitiva, afectiva y conductual).

Por último, según Portolés y González (2015), el engagement tiene una relación positiva con los resultados académicos de los adolescentes. También, el bienestar psicológico influye de manera favorable en el rendimiento académico, en la eficacia y en las calificaciones académicas.

\section{CONCLUSIONES/DISCUSIÓN}

Tras el análisis de las publicaciones, se ha comprobado que, en el contexto académico de la sociedad moderna, los adolescentes no siempre perciben sensaciones negativas, sino que también experimentan sensaciones de interés, entusiasmo, absorción, dedicación y compromiso (Schaufeli y Bakker, 2004), desarrollando las habilidades y dimensiones del engagement académico.

El engagement académico, según Schaufeli et al. (2002) y Serrano y Andreu (2016), tiene influencia en los adolescentes, ya que la atención emocional de los 
adolescentes tiene una correlación positiva con el engagement académico. Así como con el estrés que perciben.

La finalidad de este estudio es conocer y analizar la investigación previa sobre la relación entre las variables de engagement e Inteligencia Emocional. Los resultados obtenidos de los estudios confirman que sí existe una correlación significativa entre ambas variables. Así como, una relación negativa entre IE y estrés, tal y como afirman Serrano y Andreu (2016).

Además, el engagement académico, según Bresó et al. (2011), Vera (2014), Extremera (2007) y Moyano (2013), se relaciona con una alta autoeficacia académica, un rendimiento académico extraordinario, inteligencia emocional y con una satisfacción académica de los estudiantes. Estas afirmaciones se han demostrado en el estudio de Serrano y Andreu (2016), ya que verifican que el engagement académico se correlaciona con el bienestar y el rendimiento. Es por este motivo que algunas investigaciones prestan atención a la relación entre el engagement académico y la inteligencia emocional (Extremera et al., 2007).

Por otra parte, Serrano y Andreu (2016) respaldan que tener un bienestar emocional es consecuencia de tener un buen manejo y control de las emociones y, tal y como confirma Fernández (2013), la IE es una habilidad para procesar la información sobre las emociones y tener control sobre ellas. Este control permite adaptarnos al ambiente que nos rodea, percibiendo un buen clima de estudio y provocando el éxito. Es decir, favoreciendo el engagement.

El interés de este estudio y de los resultados obtenidos se centran en analizar la relación entre el engagement académico y la Inteligencia Emocional en los adolescentes, con la finalidad de conocer la influencia del desarrollo de habilidades de IE en el compromiso de los estudiantes y viceversa, así como sus consecuencias.

Para futuras líneas de investigación sería conveniente analizar trabajos que centren la atención en habilidades más concretas de la Inteligencia Emocional, como la empatía, responsabilidad, asertividad, automotivación, etc., con el objetivo de conocer si influye o no en el engagement. Además de, centrar la atención en un rango de edad más acotado, puesto que la relación entre las variables puede modificarse de un curso académico a otro.

En el presente trabajo se pueden destacar las siguientes limitaciones: 1) escasa información sobre la relación entre el engagement e Inteligencia Emocional en relación a los adolescentes, 2) extensa amplitud de trabajos basados en una bibliografía anterior a 2007.

Finalmente, hay que prestar especial atención a la relación entre el engagement académico y la inteligencia emocional (Extremera et al., 2007), para conseguir que los estudiantes adolecentes desarrollen habilidades propias de la IE y, como consecuencia, estén satisfechos académicamente. 


\section{REFERENCIAS}

Bresó, E., Schaudeli, W.B., y Salanova, M. (2011). Can a self-efficacy-based intervention decrease burnout, increase engagement, and enhance performance? A quasi experimental study. Higher Education, 61, 339-355.

Extremera, N., Durán, A., y Rey, L. (2007). Inteligencia emocional y su relación con los niveles de burnout, engagement y estrés en estudiantes universitario<s. Revista de Educación, 342, 239-256.

Fernández, M. (2013). La inteligencia emocional. Revista de Clasesgistoria, (7), 1-12.

García, E., Lorenzino, L., y Gray, S. (2019). Not Academic Enough? Enjoyment of Physical Education and the Arts nad School Engagement in Early and Middle Adolescence. Retos, 35, 301-309.

Gómez-Fraguela, J.A., Fernández, N., Romero, E., y Luengo, A. (2008). El botellón y el consumo de alcohol y otras drogas en la juventud. Trastornos Adictivos, 20(2), 211-217.

Gutiérrez, M., Tomás, J.M., Barrica, J.M., y Romero, I. (2017). Influencia del clima motivacional en clase sobre el compromiso escolar de los adolescentes y su logro académico. Enseñanza and Teaching, 35(1), 21-37.

Moyano, N., y Riaño, D. (2013). Burnout escolar en adolecsnetes espaoles: Adaptación y validación del School Burnout Inventory. Ansiedad y estrés, 19, 95-103.

Palacio, S., Caballero, C., González, O., Gravini, M., y Contreras, K. (2012). Relación del burnout y las estrategias de afrontamiento con el rendimiento académico en estudiantes universitarios. Universytas Psychological, 11(2), 178-186.

Portilés, A., y González, J. (2015). Rendimiento académico y correspondencias con indicadores de salud física y psicológica. Sportis: Revista Técnico Científica del Deporte Escolar, Educación Física y Psicomotricidad, 1(2), 164-181.

Rey, L., Extremera, N., y Pena, M. (2011). Perceived amotional intelligence, self-esteem and life satisfaction in adolescents. Psychosocial Intervention, 20(2), 227-234. doi: 10.5093/in202011v20n2a10

Schaufeli, W., y Bakker, A. (2004). Job demands, job resources, and relationship with burnout and engagement: a multisample study. Journal of Organizational Behavior, 25(3), 293.

Schaufeli, W.B., Salanova, M., González-Romá, V., y Bakker, A. (2002). The measurement of burnout and engagement: A confirmatory factor analytic approach. Journal of Happiness Studies, 3, 71-92. doi: 10.1023/A:1015630930326

Serrano, C., y Andreu, Y. (2016). Inteligencia emocional percibida, bienestar subjetivo, estrés percibido, engagement y rendimiento académico en adolescentes. Revista de Psicodidáctica, 21(2), 357-374.

Tomás, J.M., Gutiérrez, M., Sancho, P., Chireac, S.M., y Romero, I. (2016). El compromiso escolar (school engagement) de los adolescentes: medida de sus dimensiones. Enseñanza and Teaching: Revista interuniversitaria de didáctica, 34(1), 119-135.

Trujillo, M.M. (2005). Orígenes, evolución y modelos de inteligencia emocional. INNOVAR, Revista de Ciencias Administrativas y Sociales, 15(25), 3-16.

Usán, P., Salavera, C., y Domper, E. (2018). ¿Cómo se interrelacionan las variables de burnout, engagement y autoeficacia académica? Un estudio con adolescentes escolares. Revista Electrónica Interuniversitaria de Formación del Profesorado, 21(2), 141-153. 
Veiga, F.H., García, F., Reeve, J., Weantzel, K., y García, O. (2015). When Adolescents with Hihgt Self-Concept Lose their Engagement in School. Revista de Psicodidáctica, 20(2), 305320.

Vera, M., Le Blanc, P., Taris, T., y Salanova, M. (2014). Patterns of engagement: the relationship between efficacy beliefs and task engagement at the individual versus collective level. Journal Applied Social Psychology, 44, 133-144.

Recibido: 10 de febrero de 2020 Recepción Modificaciones: 08 de marzo de 2020 Aceptado: 10 de marzo de 2020 